\title{
Splenectomy with endotherapy in non-cirrhotic portal fibrosis related portal hypertension: Can it be an alternative to proximal spleno-renal shunt?
}

\author{
Sundeep Singh Saluja ${ }^{1}$, Ajay Kumar ${ }^{2}$, Hari Govind ${ }^{1}$, Vaibhav Kumar Varshney ${ }^{1}$, \\ Rahul Khullar ${ }^{1}$, and Pramod Kumar Mishra ${ }^{1}$
}

Departments of ${ }^{1}$ Gastrointestinal Surgery and ${ }^{2}$ Gastromedicine, Govind Ballabh Pant Institute of Post Graduate Medical Education and Research, New Delhi, India

\begin{abstract}
Backgrounds/Aims: Proximal splenorenal shunt (PSRS) is usually done in symptomatic non-cirrhotic portal fibrosis (NCPF). The outcomes of splenectomy with endotherapy in non-bleeder NCPF patients has not been well studied. We here by aimed to study the post-surgical outcomes on short and long-term basis between PSRS and splenectomy among non-bleeder NCPF patients. Methods: The consecutive non-bleeder NCPF patients whom underwent either splenectomy or PSRS from 2008 to 2016 were enrolled. The patients were followed up post-surgery clinically and biochemical investigations, Doppler ultrasound and upper gastrointestinal endoscopy were done as required. The peri-operative parameters compared were operative time, blood loss, hospital stay and morbidity. The long-term outcome measures compared were incidence of portal hypertension (PHTN) related bleed, change in grade of varices, shunt patency, shunt complications and thrombosis of spleno-portal axis. Results: Among 40 patients with non-bleeder status, 24 underwent splenectomy and 16 underwent PSRS. The baseline characteristics including indication of surgery, biochemical investigations and grade of varices were comparable between PSRS and splenectomy. The peri-operative morbidity was not significantly different between two groups. The median follow up duration was 42 months (12-72 months), the decrement in grade of varices was significantly higher in PSRS group $(p=0.03)$, symptomatic PHTN related UGIB was non-significant between PSRS and splenectomy $(p=0.5)$. In PSRS group, $3(18.3 \%)$ patients had shunt thrombosis $(n=1)$ \& encephalopathy $(n=2)$ while in splenectomy group two patients developed thrombosis of splenoportal axis. Conclusions: Splenectomy with endotherapy is alternative to PSRS in non-bleeder NCPF patients with indications for surgery. (Ann Hepatobiliary Pancreat Surg 2020;24:168-173)
\end{abstract}

Key Words: Non-cirrhotic portal fibrosis; GI bleed; Spleno-renal shunt; Splenectomy; Endotherapy

\section{INTRODUCTION}

Non-cirrhotic portal hypertension ( $\mathrm{NCPH})$ is a heterogeneous group of disorders characterized by portal hypertension but normal liver function and morphology. ${ }^{1,2}$ Noncirrhotic portal fibrosis (NCPF) and Extrahepatic portal venous obstruction (EHPVO) are two major causes of $\mathrm{NCPH}$. The major cause of mortality and morbidity in patients with NCPH are variceal bleeding, symptomatic hypersplenism, pain due to enlarged spleen and portal biliopathy. ${ }^{3-5}$

The frequency of NCPF among patients of PHTN in studies from India ranges from $0.9 \%$ to $46.7 \%{ }^{4}$ The NCPF is a disease of unknown etiology characterized by periportal fibrosis resulting in development of pre-sinusoidal portal hypertension (PHTN) with normal liver morphology, preserved liver functions and patent portal, splenic, hepatic veins and inferior vena cava. ${ }^{6}$ The most common presenting symptoms in patients with NCPF are variceal upper gastrointestinal bleed (UGIB) (72\%) and symptomatic (pain, transfusion-dependent anaemia, splenic infarct) splenomegaly $(12 \%){ }^{4}$

Endoscopic therapy alone, as a part of primary prophylaxis, requires regular frequent long term follow up and

Received: November 12, 2019; Revised: February 1, 2020; Accepted: March 4, 2020

Corresponding author: Sundeep Singh Saluja

Department of Gastrointestinal Surgery, Govind Ballabh Pant Institute of Post Graduate Medical Education and Research, 1, Jawahar Lal Nehru Marg, New Delhi 110002, India

Tel: +91-9718599259, E-mail: sundeepsaluja@yahoo.co.in

Copyright (C) 2020 by The Korean Association of Hepato-Biliary-Pancreatic Surgery

This is an Open Access article distributed under the terms of the Creative Commons Attribution Non-Commercial License (http://creativecommons.org/ licenses/by-nc/4.0) which permits unrestricted non-commercial use, distribution, and reproduction in any medium, provided the original work is properly cited. Annals of Hepato-Biliary-Pancreatic Surgery - pISSN: 2508-5778 - elSSN: 2508-5859 
moreover, it does not treat underlying symptomatic hypersplenism, portal biliopathy and pain due to enlarged spleen causing poor quality of life. ${ }^{7}$ Patients on endoscopic management can develop other complications of PHTN portal biliopathy and bleeding due to ectopic varices and portal hypertensive gastropathy (PHG).

The surgical options in NCPF patients include portosystemic shunts or oesophagogastric devascularization with splenectomy. Pal et al. ${ }^{8}$ raised the argument against shunt surgery as the first line management in non-bleeder NCPF where he reported $47 \%$ morbidity following it. Hence, splenectomy can be used as an alternative to shunt surgery in non-bleeder NCPF patients and can be combined with endoscopic therapy especially if high risk varices are present.

There are no studies comparing the outcomes of shunt surgery versus splenectomy (with or without endotherapy) in non-bleeder NCPF patients. In present study, we have compared immediate and long-term outcomes between proximal spleno-renal shunt (PSRS) and splenectomy with endoscopic therapy in non-bleeder NCPF patients with hypersplenism and symptomatic splenomegaly.

\section{MATERIALS AND METHODS}

The study was carried out in the department of Gastrointestinal surgery and Gastroenterology based on a prospectively maintained departmental database of PHTN patients. The records of all patients with diagnosis of NCPF who were managed surgically from January 2008 to December 2016 were reviewed. Total of 78 patients with NCPF were managed over the study period, 40 NCPF patients with non-bleeder status were enrolled in the study, $38 \mathrm{NCPF}$ patients whom had history of UGI Bleed related to PHTN were excluded from study. The work has been reported in line with the STROCSS criteria. ${ }^{9}$

\section{Baseline evaluation}

All patients with portal hypertension underwent detailed history and physical examination. The investigations done were hemogram, liver function test, renal function test and serology for hepatitis B and C virus, Ultrasound (US) of abdomen with Doppler imaging and Upper Gastrointestinal endoscopy (UGIE). The findings noted on US with Doppler were liver size, surface and its echotexture, hepatic vein and inferior vena cava patency, portal vein patency, flow velocity and its diameter, splenic vein patency and diameter, collaterals and their location, spleen size and ascites. The computed tomography (CT) of abdomen was done when there was any suspicion of cirrhosis on US for detail study of liver morphology. The UGIE was done under local anesthesia for varices (esophageal, gastric and ectopic) and PHG.

The pre-operative diagnosis of NCPF was made by combination of clinical findings, biochemical, radiological and endoscopy investigations. The indications of surgery in non-bleeders NCPF patients were symptomatic hypersplenism and symptomatic (painful) splenomegaly causing poor quality of life.

\section{Management protocol}

Patients underwent either proximal spleno-renal shunt (PSRS) or splenectomy. The patients undergoing splenectomy alone underwent prophylactic endoscopic band ligation if they had high risk varices prior or post-surgery. Intra-operative liver biopsy was done in all patients to rule out cirrhosis. The technique used for PSRS was as described in literature. ${ }^{10}$ The end to side spleno-renal shunt was performed with 6-0 polypropylene (Double ended, Round bodied $11 \mathrm{~mm}$ needle). Post-operatively patients were not given any anticoagulants.

The patients were followed post discharge every week for first month and then every 3 months for first year then every 6 months for next 3 years and yearly thereafter. On follow up visits patients were evaluated clinically for shunt related complications and biochemical investigations, ultrasound abdomen with Doppler, UGI Endoscopy were done and CT abdomen with portography if required.

\section{Baseline characteristics}

The baseline characteristics including age, sex, haemoglobin, total leucocyte count, platelets counts, h/o jaundice, symptomatic splenomegaly and hypersplenism and the grade of varices were compared among the two groups.

\section{Outcome parameters}

The immediate outcome parameters measured and compared were duration of surgery, blood loss, length of hospital stay, mortality, and morbidity. The long-term outcome parameters measured and compared were portal hy- 
pertension related bleed, decrement in grade of varices, shunt related complications (encephalopathy, nephropathy and myleopathy), shunt patency, and thrombosis of spleno-portal axis.

\section{Statistical analysis}

Categorical variables and continuous variables were analysed by Fisher's exact test/Chi square test and MannWhitney's test respectively. A $p$-value less than 0.05 was considered statistically significant. All statistical analysis was performed using SPSS for Windows 22.1 software (SPSS, Chicago, Illinois, USA).

\section{RESULTS}

Out of 40 non-bleeder NCPF patients, 16 underwent PSRS (Group 1) and 24 underwent splenectomy alone (Group 2). The median age was 27.9 years (8-55 years) and $33(82.5 \%)$ were females. The median duration of symptoms at time of presentation was 3 years (range 1-14 years). The baseline characteristics, indications of surgery and endoscopy findings (large grade esophageal varices PSRS $\mathrm{n}=7 / 16,43 \%$ and splenectomy group $\mathrm{n}=8 / 24,33.3 \%$, $p=0.2$ ) were comparable between two groups (Table 1).

\section{Outcomes (Table 2 and Table 3)}

The median duration of surgery (PSRS- $215 \mathrm{~min}$ [180-260 $\mathrm{min}]$, Splenectomy- $95 \mathrm{~min}$ [60-120 $\mathrm{min}]$; $p=0.04$ ) and blood loss (PSRS- $375 \mathrm{ml}$ [180-600 ml],
Splenectomy-200 $\mathrm{ml}[30-500 \mathrm{ml}] ; p=0.03)$ were significantly higher in PSRS group as compared to splenectomy group. The median duration of hospital stay was similar in both groups (PSRS- 5 days [3-25 days], Splenectomy 5 days [3-6 days], $p=0.4$ ). There was no operative mortality and morbidity rate was $16 \%$ in PSRS $11 \%$ in splenectomy group ( $p=0.32$ ).

The median duration of follow up was 42 months (1272 months). Overall decrease in the size of varices was seen in $11 / 16(68.7 \%)$ patients of PSRS group and in $8 / 24$ $(33.3 \%)$ patients of splenectomy group $(p=0.028)$. The UGIB related to PHTN occurred in $2 / 16$ patients in the PSRS group $(6.2 \%)$ and $1 / 24(4.1 \%)$ patient in splenectomy group ( $p=0.55$ ). The post-shunt encephalopathy was seen in one patient after PSRS while none of the patients in the splenectomy group had encephalopathy. Two patients in splenectomy group developed thrombosis of splenoportal axis. None of these patients had rebleed till last follow up.

Overall hypersplenism improved in both groups and median hemoglobin increased from 8 to $9.5 \mathrm{mg} / \mathrm{dl}$, leukocyte count from 2500 to $8200 \mathrm{cu} / \mathrm{mm}^{3}$ and platelet count from 49000 to $411000 \mathrm{cu} / \mathrm{mm}^{3}$.

Two patients in splenectomy group and one patient in PSRS group died in long term follow up probably cause unrelated to treatment related complication. In splenectomy group one patient at 3.5 year follow up succumbed to febrile illness while other patient developed chronic renal failure at 1 year follow up, while patient with PSRS

Table 1. Comparison of baseline characteristics between PSRS and splenectomy group

\begin{tabular}{lccc}
\hline \multicolumn{1}{c}{ Parameters } & PSRS group $(\mathrm{n}=16)$ & Splenectomy group $(\mathrm{n}=24)$ & $p$-value \\
\hline Age (years) Median (Range) & $25.5(8-55)$ & $25(17-52)$ & 0.881 \\
Gender (Male:Female) & $4: 12$ & $3: 21$ & 0.308 \\
Symptomatic Hypersplenism; n (\%) & $12(75)$ & $18(75)$ & 1.000 \\
Symptomatic Splenomegaly; n (\%) & $4(25)$ & $6(25)$ & 1.000 \\
Jaundice; n (\%) & $4(25)$ & $7(29)$ & 0.772 \\
Grade of varices & 7 & 16 & 0.151 \\
Small & 9 & 8 & 0.304 \\
Large & $9.45(3-13.2)$ & $8.05(4.4-13.1)$ & 0.838 \\
Hemoglobin; Median (range) (gm/dl) & $2400(1100-9300)$ & $2650(1000-13000)$ & 0.795 \\
TLC; Median (range) (cells/dl) & $43000(22000-160000)$ & $47000(5000-140000)$ & 0.070 \\
Platelet; Median(range) (cells/dl) & $0.7(0.4-2.3)$ & $1.30(0.4-3.0)$ & 0.126 \\
Bilirubin; Median(range) mg/dl & $121(59-280)$ & $146(82-319)$ & \\
ALP; Median (range) IU/L & & & \\
\hline
\end{tabular}

TLC, total leucocyte count; ALP, alkaline phosphatase 
Table 2. Comparison of peri-operative outcomes

\begin{tabular}{lccc}
\hline \multicolumn{1}{c}{ Outcome parameters } & PSRS $(\mathrm{n}=16)$ & Splenectomy (n=24) & $p$-value \\
\hline Duration of surgery (min) Median (Range) & $215(180-260)$ & $95(60-120)$ & 0.04 \\
Blood loss (ml) Median (Range) & $375(180-600)$ & $200(30-500)$ & 0.03 \\
Hospital stay (days) Median (Range) & $5(3-25)$ & $5(3-6)$ & 0.45 \\
Morbidity & $16 \%$ & $11 \%$ & 0.32 \\
Clavien Dindo Complication ( $\leq \mathrm{II})$ & 3 & 3 & 0.38 \\
\hline
\end{tabular}

Table 3. Comparison of post-operative outcomes in both groups

\begin{tabular}{lccc}
\multicolumn{1}{c}{ Outcome parameters } & PSRS $(\mathrm{n}=16)$ & Splenectomy $(\mathrm{n}=24)$ & $p$-value \\
\hline UGI bleed n (\%) & $2(12.5)$ & $1(4.2)$ & 0.553 \\
Decrement in grade of varices n (\%) & $11(68.7)$ & $8(33.3)$ & 0.028 \\
Status of Shunt & & NA & - \\
Patent $\mathrm{n}(\%)$ & $15(93.75)$ & & \\
Thrombosed n (\%) & $1(6.25)$ & 2 & 0.159 \\
Spleno-portal axis thrombosis & 0 & $10.5(5.8-14.6)$ & 0.699 \\
Hemoglobin; Median (range) (g/dl) & $9.4(5.8-12.8)$ & $8200(5000-12300)$ & 0.052 \\
TLC; Median (range) (cells/dl) & $8300(5600-12300)$ & $242500(62000-600000)$ \\
Platelet; Median (range) (cells/dl) & $340000(120000-632000)$ & $0.55(0.2-1.4)$ & 0.835 \\
Bilirubin; Median (range) mg/dl & $0.6(0.2-1.4)$ & $226.0(72-535)$ & 0.029 \\
ALP; Median (range) IU/L & $152.5(73-296)$ & $0(0)$ & 0.154 \\
Encephalopathy n (\%) & $2(12.5)$ & & \\
\hline
\end{tabular}

UGI, upper gastrointestinal; TLC, total leucocyte count; ALP, alkaline phosphatase

succumbed to febrile illness.

\section{DISCUSSION}

Proximal spleno-renal shunt has been shown to be effective in not only preventing variceal bleeding but also in managing associated hypersplenism and portal biliopathy. ${ }^{11}$ Shunt surgery is one time treatment and useful if patient does not have access to endotherapy especially at time of acute UGI bleeding. The beneficial effects of shunt surgery are mitigated when shunt get blocked. Mishra et al. ${ }^{10}$ reported that $25 \%(3 / 12)$ of NCPF patients developed the shunt blockage during follow up. In present study shunt blockage rate was $1 / 16(6.25 \%)$, the low rate may be due to the fact that we did not used CT portography routinely in all patients on follow up to assess shunt patency.

Splenectomy alone is considered as sub-optimal treatment in NCPF, post splenectomy increase risk of thrombosis of spleno-portal venous axis is there and it can result in increased risk of PHTN related bleed. However, with the hypothesis that removal of large spleen in these patients decreases portal pressure to some extent and over the period of time will lead to decrease in grade of varices made us to perform comparative study with PSRS.

In present study two groups were comparable in terms of age and sex distribution, duration of symptoms, indications for the surgery and severity of PHTN as shown by grade of varices at time of presentation. In either group there was no immediate post-operative mortality and morbidity rates were comparable. This is in accordance with previously published studies. ${ }^{12}$ Overall, splenectomy alone has advantages in terms of shorter operative time and lower blood loss with similar long-term outcomes compared to PSRS in Non-Cirrhotic Portal Fibrosis patients without gastrointestinal bleed.

The present study had fairly long follow-up, post-surgery median duration of follow up was 4 years in both groups. There was significant improvement in hypersplenism after surgery in both the groups. The similar outcome was observed in previously published study though the number of patients who underwent splenectomy were too small for drawing any inference. ${ }^{12}$ The decrement in grade of varices was significantly better in patients undergoing PSRS 11/16 (68.7\%) than splenectomy 8 (33.3\%), however bleeding rates related to PHTN however was not significantly different among two groups (PSRS 12.5\% vs. 
Splenectomy $4.2 \%$ ). This can be explained by regular endoscopic surveillance done in present study during the follow up.

The other long term complication with PSRS is hepatic encephalopathy and rarely nephropathy and myelopathy can also occur both of these complications are unpredictable and irreversible. ${ }^{13-15}$ The overt hepatic encephalopathy rate $(12.5 \%)$ in present study was comparable to previous study by $\mathrm{Pal}$ et al. ${ }^{8}$ where $13 \%$ patients developed hepatic encephalopathy. The reason for low rate of hepatic encephalopathy in NCPF than cirrhosis post shunt surgery is better reserve of hepatic functions especially in non-bleeder NCPF patients. In the present study two patients post PSRS developed overt hepatic encephalopathy and it could be managed with oral lactulose. In present study post-PSRS we did not encountered nephropathy or myelopathy. In previous study by Pal et al. ${ }^{8}$ post-PSRS 4/41 (9.7\%) NCPF patients developed nephropathy, 2 patients needed long-term hemo-dialysis and one patient died. The cause of nephropathy post PSRS in NCPF is not clear and it may be either due to underlying aetiology or consequence of shunt surgery.

On the other hand, splenectomy alone is considered suboptimal since it does not mitigate PHTN totally. However, Ohnishi et al. ${ }^{16}$ has suggested that at an early stage of the disease, portal veins may be dilated without increased portal pressure, primarily non-bleeder status. In this subclass of patients, doing PSRS may not help, but performing splenectomy alone will decrease pain due to splenomegaly \& treat hypersplenism and prevent long term complications of PSRS. Similar approach has been attempted by Belhamidi et al. ${ }^{17}$ with success; however their numbers are very small.

The major limitation of our study is that it is nonrandomized retrospective analysis, other is minimal hepatic encephalopathy (MHE) was not assessed.

To conclude, splenectomy alone decreases portal pressure to some extent and as over the period the increase in grade of varices was not noted. Hence, splenectomy combined with endoscopic surveillance is an alternative to PSRS to treat hypersplenism and symptomatic splenomegaly in non-bleeder NCPF patients.

\section{CONFLICT OF INTEREST}

None of the authors have any potential conflicts to disclose.

\section{ORCID}

\author{
Sundeep Singh Saluja: \\ https://orcid.org/0000-0003-2733-7204
}

Ajay Kumar: https://orcid.org/0000-0002-5877-4610

Hari Govind: https://orcid.org/0000-0001-7908-6086

Vaibhav Kumar Varshney:

https://orcid.org/0000-0003-1771-2787

Rahul Khullar: https://orcid.org/0000-0002-5246-2915

Pramod Kumar Mishra:

https://orcid.org/0000-0003-0005-4044

\section{AUTHOR CONTRIBUTIONS}

Conceptualization: SSS, AK, PKM. Data curation: SSS, HG, VKV, RK. Formal analysis: SSS, AK, HG, VKV, RK, PKM. Methodology: SSS, AK, PKM. Project administration: SSS, AK, PKM. Visualization: SSS, AK, PKM. Writing - original draft: HG, VKV, RK. Writing - review \& editing: SSS, AK, VKV, PKM.

\section{REFERENCES}

1. Khanna R, Sarin SK. Non-cirrhotic portal hypertension - diagnosis and management. J Hepatol 2014;60:421-441.

2. Schouten JN, Garcia-Pagan JC, Valla DC, Janssen HL. Idiopathic noncirrhotic portal hypertension. Hepatology 2011;54:1071-1081.

3. Sarin SK, Agarwal SR. Extrahepatic portal vein obstruction. Semin Liver Dis 2002;22:43-58.

4. Dhiman RK, Chawla Y, Vasishta RK, Kakkar N, Dilawari JB, Trehan MS, et al. Non-cirrhotic portal fibrosis (idiopathic portal hypertension): experience with 151 patients and a review of the literature. J Gastroenterol Hepatol 2002;17:6-16.

5. Schouten JN, Nevens F, Hansen B, Laleman W, van den Born M, Komuta M, et al. Idiopathic noncirrhotic portal hypertension is associated with poor survival: results of a long-term cohort study. Aliment Pharmacol Ther 2012;35:1424-1433.

6. Sarin SK, Kumar A. Noncirrhotic portal hypertension. Clin Liver Dis 2006;10:627-651, $\mathrm{x}$.

7. de Franchis R; Baveno VI Faculty. Expanding consensus in portal hypertension: report of the Baveno VI Consensus Workshop: stratifying risk and individualizing care for portal hypertension. J Hepatol 2015;63:743-752.

8. Pal S, Radhakrishna P, Sahni P, Pande GK, Nundy S, Chattopadhyay TK. Prophylactic surgery in non-cirrhotic portal fibrosis: is it worthwhile? Indian J Gastroenterol 2005;24:239242. 
9. Agha RA, Borrelli MR, Vella-Baldacchino M, Thavayogan R, Orgill DP; STROCSS Group. The STROCSS statement: Strengthening the Reporting of Cohort Studies in Surgery. Int J Surg 2017;46:198-202.

10. Mishra PK, Patil NS, Saluja S, Narang P, Solanki N, Varshney V. High patency of proximal splenorenal shunt: a myth or reality ? - a prospective cohort study. Int J Surg 2016;27:82-87.

11. Prasad AS, Gupta S, Kohli V, Pande GK, Sahni P, Nundy S. Proximal splenorenal shunts for extrahepatic portal venous obstruction in children. Ann Surg 1994;219:193-196.

12. Rajalingam R, Javed A, Sharma D, Sakhuja P, Singh S, Nag HH, et al. Management of hypersplenism in non-cirrhotic portal hypertension: a surgical series. Hepatobiliary Pancreat Dis Int 2012;11:165-171.

13. Sarin SK, Nundy S. Subclinical encephalopathy after portosystemic shunts in patients with non-cirrhotic portal fibrosis. Liver
1985;5:142-146.

14. Kumar A, Bhuyan UN, Nundy S. Glomerulonephritis complicating non-cirrhotic portal fibrosis. J Gastroenterol Hepatol 1989;4 Suppl 1:271-275.

15. Dash SC, Bhuyan UN, Dinda AK, Saxena S, Agarwal SK, Tiwari $\mathrm{SC}$, et al. Increased incidence of glomerulonephritis following spleno-renal shunt surgery in non-cirrhotic portal fibrosis. Kidney Int 1997;52:482-485.

16. Ohnishi K, Saito M, Sato S, Terabayashi H, Iida S, Nomura F, et al. Portal hemodynamics in idiopathic portal hypertension (Banti's syndrome). Comparison with chronic persistent hepatitis and normal subjects. Gastroenterology 1987;92:751-758.

17. Belhamidi MS, Hammi SE, Bouzroud M, Benmoussa M, Ali AA, Bounaim A. [Role of splenectomy in the treatment of non-cirrhotic portal hypertension: about 3 cases]. Pan Afr Med J 2017;28:84. French. 